\title{
Das Ganze und die Teile
}

\section{Die Deutsche Gesellschaft für Soziale Arbeit diskutierte über Generalisierung und Spezialisierung in Ausbildung und Beruf}

\author{
Albert Mühlum
}

Prof. Dr. Albert Mühlum war bis zu seiner Pensionierung Hochschullehrer für Sozialpolitik und

Sozialarbeitswissenschaft an der Fachhochschule Heidelberg und Lehrbeauftragter der Universität Heidelberg. Er war lange Jahre Vorstandsmitglied der Deutschen Gesellschaft für Soziale Arbeit e. V. E-Mail a.muehlum@t-online.de
Die Soziale Arbeit in Deutschland hatte schon immer mit Fragen von Einheit und Differenzierung zu kämpfen. Die lange Zeit geltende Zweiteilung in Sozialarbeit und Sozialpädagogik ist ein Beispiel dafür. Mit der Bologna-Reform der Hochschulausbildung und der Neuordnung des Weiterbildungsmarktes stellen sich die Fragen von Generalisierung und Spezialisierung in der Sozialen Arbeit erneut.

Ein kühner Bogen: »Das Ganze und seine Teile « ist eine Herausforderung, die selbst in der Philosophie nicht abschließend gemeistert wurde. Die Eingrenzung auf Generalisierung und Spezialisierung Sozialer Arbeit schien da schon eher bewältigbar zu sein.

Angemessen, ja notwendig, ist das Thema allemal, vertritt die Sozialarbeit doch eine Ganzheitsperspektive, die historisch als »Person-in-der-Situation-Konfiguration « (Gordon Hamilton) und aktuell als "Person-in-Environment « (Karls/Wandrei) zum Kern des sozialberuflichen Selbstverständnisses zählt, während gleichzeitig eine immer stärkere Ausdifferenzierung bis hin zur Etablierung von »Fachsozialarbeit « stattfindet. Im Flyer zur Jahrestagung 2010 der Deutschen Gesellschaft für Soziale Arbeit hieß es dazu:

"Spätestens mit der Einführung gestufter Studiengänge bei den sozialen Berufen und einer Neuordnung des Weiterbildungsmarktes bilden sich nach der Konvergenz von Sozialpädagogik und Sozialarbeit neue Formen der Spezialisierung im Handlungsfeld der Sozialen Arbeit und ihrer benachbarten Professionen heraus. So entstanden infolge des Bolognaprozesses eine Vielzahl hoch spezialisierter Masterstudiengänge, bei denen eine fachwissenschaftliche Verankerung in der Sozialen Arbeit kaum noch zu erkennen ist ... Neue Formen der Generalisierung entstehen ebenso wie neue Formen der Spezialisierung. Obwohl dieser Prozess in Ausbildung und Praxis teilweise zu hefti- gen Kontroversen geführt hat, wurde dieser in der theoretischen Reflexion bisher kaum thematisiert. «

Grund genug also, sich in der wissenschaftlichen Gemeinschaft, die zum großen Teil in der Deutschen Gesellschaft für Soziale Arbeit organisiert ist, damit auseinanderzusetzen.

Der Rahmen vor Ort war beeindruckend. Der Rektor der gastgebenden Evangelischen Hochschule Freiburg, Prof. Dr. Reiner Marquard, konnte rund 200 Teilnehmerinnen und Teilnehmer begrüßen, die zum überwiegenden Teil selbst zur Professorenschaft zählen. Fast 60 Referentinnen und Referenten standen für drei Hauptvorträge, neun zweistufige Panels mit jeweils bis zu vier Einzelpräsentationen sowie für zusätzliche Ad-hocGruppen und das Abschlusspodium zur Verfügung.

Schon bei der Eröffnung wurde auf das Bemühen um »Ganzheit « in einem spezifischen Sinne hingewiesen, da in einer Vorkonferenz wichtige Akteure der Sozialen Arbeit erstmals gemeinsam tagten: die Deutsche Gesellschaft für Soziale Arbeit als Veranstalter, der Fachbereichstag Soziale Arbeit als nationale Repräsentanz der Fachbereiche und Fakultäten Soziale Arbeit der staatlichen und kirchlichen Hochschulen in der Bundesrepublik Deutschland), die Sektion Sozialpädagogik der Deutschen Gesellschaft für Erziehungswissenschaft, der Deutscher Berufsverband für Soziale Arbeit und der Deutsche Verein für öffentliche und private Fürsorge. Diese Institutionen waren später auch auf dem Abschlusspodium prominent vertreten.

In der Begrüßung wurde des im letzten Jahr verstorbenen Kollegen Prof. Dr. Konrad Maier gedacht, der mit der Gründung und langjährigen Leitung des bis dato größten Forschungsinstituts an Hochschulen des Sozialwesens wesentlich zur Entwicklung der Profession und Disziplin beigetragen hat, zuletzt auch zur Einrichtung eines konsekutiven for- 
schungsorientierten Masterstudiengangs an der Evangelischen Hochschule Freiburg. Der Rektor verstand es, die Hommage an einen verdienten Wissenschaftler mit der Zielsetzung des Kongresses zu verknüpfen: Bei aller Divergenz möge die Tagung dazu beitragen, »das kostbare Gut der Sozialen Arbeit (!) gemeinsam zu fördern «.

Für die Deutsche Gesellschaft für Soziale Arbeit griff der Vorsitzende, Prof. Dr. Herbert Effinger, den Gedanken auf. Das Ganze und seine Teile versuchte er in Metaphern wie »Puzzle « und »Baum « zu fassen, die alle aus Elementen bestehen aber höchst unterschiedlich in Komplexität und Dynamik. Sozialarbeit und Sozialpädagogik seien in diesem Bild vielleicht eher zwei Bäumchen, die langsam ineinander wachsen, wobei die Ausdifferenzierung der Deutschen Gesellschaft für Soziale Arbeit selbst - in mittlerweile zehn Sektoren und Fachgruppen - den Prozess des Wachsens und Veränderns widerspiegle. Schließlich leitete Prof. Dr. umgeschichtet wurden, die »Internationalität « jedoch lag somit allein bei den deutschsprachigen Nachbarländern, was auch als Hinweis auf die schwache internationale Vernetzung der deutschen Sozialen Arbeit gedeutet werden kann. Dessen ungeachtet waren die Vorträge sehr informativ und in Teilen höchst unterhaltsam.

\section{Vortrag I: »Generalisierung und Spezialisierung Sozialer Arbeit in der Schweiz «}

Daniel Gredig und Stefan Schnurr (Fachhochschule Nordwestschweiz) referierten in vier Teilen:

- Entwicklung des Bildungssystems Soziale Arbeit in der Schweiz: von der Fachausbildung zur Akademisierung - und zurück

- der Bolognaprozess: die Verständigung auf ein generalistisches Master-Studium

\section{"Theorie, Methode, Technik und Praxis müssen eine Einheit bilden- wenn auch mit Widersprüchen "}

Björn Kraus als Dekan der Evangelischen Hochschule Freiburg zur Organisation der Tagung und den thematischen Schwerpunkten über, wohl wissend, dass Generalisierung und Spezialisierung keine neuen Themen sind, dass sie aber immer wieder grundsätzlich und in konkreten Ausprägungen zu entscheiden und zu gestalten sind, wie er am Beispiel der Stufenabschlüsse nach Bologna illustrierte.

Der » internationale Maßstab « sollte in drei Hauptvorträgen an den Beispielen Großbritannien, Schweiz und Österreich deutlich werden. Daher war es eine herbe Enttäuschung, dass der mit besonderer Spannung erwartete Beitrag von Lena Dominelli (Durham University, Großbritannien) über »International challenges to social work education « wegen Erkrankung der Referentin entfiel. Zwar entstand wegen des dichten Programms keine Lücke, da andere Programmpunkte
- die Weiterbildung: eine florierende Landschaft von Angeboten zur Weiterqualifikation

- Konklusion: Generalisierung und Spezialisierung Sozialer Arbeit in der Schweiz

In einhundert Jahren entstand in der Schweiz ein diversifiziertes System der sozialen Ausbildung mit Abschlüssen auf vier unterschiedlichen Stufen; zuletzt ein Fachhochschulgesetz (1995) mit vierfachem Leistungsauftrag: Lehre, Forschung und Entwicklung, Weiterbildung, Dienstleistungserbringung. Seit 2006 erfolgte eine Verdichtung auf sieben Fachhochschulen in der Schweiz mit den akademischen Merkmalen: Tertiärstufe (Level ISCED 5a) mit Zugang zur Promotion (Level ISCED 6).

Nach Bologna wurden systemgerecht dreistufige Studiengänge eingerichtet:

- Bachelor-Studien an Universitäten und Fachhochschulen ab 2005
- Master-Studien an Universitäten und Fachhochschulen ab 2008

- Promotion an Universitäten, teilweise Einführung von Promotionsstudien

Bachelor-Studien umfassen 180 ECTSCredits (nach dem European Credit Transfer and Accumulation System); Master-Studien an Universitäten können 90 oder 120 ECTS-Credits, jene an Fachhochschulen dürfen jedoch höchstens 90 ECTS-Credits umfassen.

Viele Aspekte sind mit der Entwicklung in Deutschland vergleichbar, beginnend mit Ausbildungsbemühungen in "Kursen « (ab 1908) bis zur Akademisierung, die allerdings erst Ende des 20. Jahrhunderts realisiert wurde. Eigenartigerweise wird der Masterabschluss mit 270 ECTS (statt 300 in Deutschland) erreicht, so dass kein nahtloser Übergang zur Promotion möglich ist. Ein weiterer Unterschied: Die schweizerischen Fachhochschulen haben sich auf generalistische Masterstudiengänge verständigt und die Spezialisierung der Weiterbildung vorbehalten.

Bachelor und Master sind in der Schweiz also generalistisch, während Spezialisierungen handlungsfeldbezogen in der Weiterbildung, theorie- und methodenbezogen in Promotionen erfolgen. Randbedingungen dafür sind $u$. a. ein breites Weiterbildungsangebot, konstruktives Zusammenwirken von Arbeitgebern und Arbeitnehmern, Angebotsformen " aus einer Hand « und Akzeptanz der Allokationsfunktion von Weiterbildungsmaßnahmen.

Vorteile in dieser Aufteilung werden darin gesehen, dass ein generalistischer Master den Übergang zur Promotion erleichtere. Ein großer Vorzug ist die Einrichtung von Forschungsprofessuren, die in den letzten Jahren zum bemerkenswerten Aufschwung der Forschung in Sozialer Arbeit führten.

Diskutiert wurden nach dem Vortrag im einzelnen: Zugangsberechtigung(en); Qualitäts- und Quantitätsfragen des Übergangs vom Bachelor zum Masterstudium und vom Master zur Promotion; Steuerung der Zulassung zum Masterstudium über Quoten oder Appelle; Erfahrungen mit Forschungsprofessuren; Anstellungschancen und Gehaltsniveau; Auswirkung der früheren Dreiteilung von Sozialarbeit, Sozialpädagogik und soziokulturelle Animation auf heutige Curricula; Praxisrelevanz und handlungsfeldbezogene Vertiefungsrichtungen. 


\section{Vortrag II: »Generalisierung und Spezialisierung Sozialer Arbeit in Österreich «}

Peter Pantucek (Fachhochschule St. Pölten, Österreich) erwähnte zunächst, seine Darstellung enthalte angesichts divergierender Auffassungen im Land wohl einen subjektiven Zungenschlag. Historisch waren in Österreich Sozialarbeiter und Sozialarbeiterin, Erzieherin und $\mathrm{Er}$ zieher getrennte Berufe auf unterschiedlichem Niveau:

- Für den Kernbereich der sozialpädagogischen Aufgaben erfolgte die Ausbildung i. d. R. in Erzieherinnenschulen oder in zweijährigen Kollegs nach dem Abitur. In den neu gegründeten pädagogischen Hochschulen werden nun auch Bachelor-Studiengänge angeboten, bei weiter bestehenden ErzieherFachschulen (Sozialpädagogen). Ein universitäres Studium der Pädagogik mit Schwerpunkt Sozialpädagogik ist möglich, spielt aber eine marginale Rolle.

- Die Sozialarbeitsausbildung erfolgte in Österreich bis zur Jahrtausendwende in Akademien (6 Semester), ab 2001 wurden die Studiengänge der Sozialarbeit in die Fachhochschulen eingegliedert und erfuhren eine beachtliche Aufwertung. Die Studienplätze sind teilweise zehnfach überbucht.

Die geringe Zahl der Plätze führt zu einem eklatanten Mangel an Sozialarbeitern im Land, mit der Folge, dass Sozialarbeitsaufgaben von Nicht-Sozialarbeitern (z. B. nach schlichten »BeraterKursen «) übernommen werden. Im »Berater-Ausbildungsmarkt « werden ungehemmt Begriffe und Konzepte der Sozialen Arbeit verwendet - ohne dies ausfüllen zu können. Daher hat für die Sozialarbeit die Abgrenzung zu anderen Ausbildungsprofilen - und damit die professionelle Identität - hohe Bedeutung.

Die Hauptprobleme in Österreich sind also: Mangel an ausreichend qualifiziertem Lehrpersonal; der künstlich generierte Mangel an graduierten Sozialarbeiterinnen und Sozialarbeitern; geringe Größe der Standorte, die keine kontinuierliche Arbeit an Forschungsschwerpunkten erlaubt und die Wahlmöglichkeiten für Studenten begrenzt.

Die Spezialisierung von Studiengängen ist uneinheitlich. In St. Pölten gilt die generalistische Orientierung für B. A. und
M. A. Die Masterstudiengänge weisen im Übrigen eine gemäßigte Spezialisierung auf: an allen Standorten werden Schwerpunkte angeboten, beispielsweise Klinische Sozialarbeit, Sozialraumorientierung, Sozialmanagement.

Im Weiterbildungsbereich können die Hochschulen Abschlüsse auf zwei Ebenen anbieten: Als Hochschul-Lehrgang mit mindestens 60 ECTS-Punkten, Abschluss z. B. "Akademische Suchtberaterin « oder als Weiterbildungs-Master mit 90 ECTS. Beide Angebote müssen durch Studiengebühren (oder Subventionen) finanziert werden. Für die Zulassung zur Promotion müssen zusätzlich zum Doktoratsprogramm bis zu 30 Semesterwochenstunden (SWS) nachgeleistet werden. Sozialarbeitswissenschaftliche Fragestellungen würden i. d. R. nicht akzeptiert oder bis zur Unkenntlichkeit verstümmelt.

Im Spannungsfeld vom Allgemeinen und Besonderen plädiert Pantucek für eine offensive Strategie: Wenn das für das Sozial- und Gesundheitswesen notwendige sozialarbeiterische Wissen wegen fehlender Sozialarbeiterinnen und Sozialarbeiter nicht zur Verfügung stehe, sollten wenigstens die Nachbarberufe einschlägiges Know-how erwerben - und zwar an den Hochschulen, beispielsweise durch die Öffnung der konsekutiven Sozialarbeits-Master für Quereinsteiger - was kontrovers diskutiert wird.

Schließlich: Die Zukunft der BachelorStudiengänge werde exemplarisch-generalistisch sein müssen - während früher die Bezugswissenschaften für das Orientierungswissen, die Methoden für den Identitätskern und die HandlungsfeldLehrveranstaltungen für die Praxisnähe sorgen sollten. Diese »Handlungsfelder « waren schon pragmatische Spezialisierungen - konnten aber weder die Breite des Feldes noch den akademischen Anspruch abdecken. Deshalb soll die Aneignung der kontextabhängigen Wissensbestände nun auch offiziell im Beruf erfolgen.

Der Referent stellte beispielhaft eine Auflistung dieses kontextspezifischen Wissens vor, dem er die kontextunabhängigen Wissensbestände gegenüberstellte, die wiederum nur exemplarisch vermittelt werden können. Mit Bezug auf Dirk Baecker wären »exemplarische Tiefenbohrungen " vorzunehmen - im Dreischritt von Methode, Theorie und Praxis, ergänzt um die Dimension Technik(en). Diese vier Elemente einer exemplarisch generalistischen Bildung seien eine widersprüchliche Einheit, die didaktisch und organisatorisch bewältigt werden müsse.

Diskutiert wurden im Anschluss an den Vortrag aus Österreich: Relation Studienplätze und Absolventen zur Bevölkerungszahl; Studiums- und Sozialarbeitsmigration; Akademie- und Hochschulabschlüsse; Qualifizierung des Lehrpersonals; Ansprüche an die Sozialarbeit; Relevanz der Praxis; Identität der Sozialen Arbeit; Fachsozialarbeit; Berufsgesetz; staatliche Anerkennung. Vor allem aber, ob die curriculare Alternative wirklich laute: »spezialisiert oder oberflächlich?«.

\section{Panels der Sektionen und Fachgruppen}

Nach den Hauptvorträgen mit Diskussionsrunden war für die Sektionen und Fachgruppen Gelegenheit an zwei Tagen in sogenannten Panels einschlägige Forschungs- und Praxisprojekte vorzustellen. Deren Rahmenthemen lauteten;

- Sektion Theorie- und Wissenschaftsentwicklung: »Wie lassen sich allgemeine und spezifische disziplinäre Inhalte Sozialer Arbeit begründen? «

- Fachgruppe Forschung: »Das Spezielle und das Allgemeine «

- Fachgruppe Promotionsförderung der Sozialen Arbeit nach FachhochschulAbschluss: »Institutionalisierte Promotionsförderung der Sozialen Arbeit «

- Sektion Klinische Sozialarbeit: »Spezialisierungsanforderungen an die Klinische Sozialarbeit in Forschung, Theoriebildung, Praxis und Ausbildung «

- Sektion Gemeinwesenarbeit: »Differenzierungen und Perspektiven «

- Fachgruppe Sozialwirtschaft: »Perspektiven im sozialen Management - Institutionen, Organisationen, Rat und Beziehungen «

- Fachgruppe Systemische Ansätze: »Der spezialisierte Generalismus der Sozialen Arbeit - systemische Perspektiven «

- Fachgruppe Politik: »Grundständige und spezifische politische Kompetenzen in der Sozialen Arbeit "

- Fachgruppe Ethik: »Ethische Fragen der Sozialarbeit im Spannungsfeld zwischen normativen Grundlagen und Handlungsanforderungen «

- Ad-hoc-Gruppen zu »Gender « und »Bezugswissenschaften der Sozialen Arbeit « 
Die Vorträge und Diskussionen in den 18 Panelsitzungen mit jeweils bis zu drei Einzelpräsentationen plus Ad-hoc-Gruppen sollen in einem Tagungsband dokumentiert werden, der in der Schriftenreihe der Deutschen Gesellschaft für Soziale Arbeit erscheinen wird. Als Beispiel seien die Beiträge zu Sektion Klinische Sozialarbeit genannt:

Teil A: Forschung und Theoriebildung (Moderation Claire Moore)

- Bettina Schuhrke (Evangelische Fachhochschule Darmstadt): Psychische Probleme bei Kindern und Jugendlichen in (teil-) stationären Erziehungshilfen - Herausforderung oder Überforderung im Hinblick auf in der Ausbildung von SozialarbeiterInnen/SozialpädagogInnen vermittelte Kompetenzen?

- Anja Terner (Fachhochschule Hannover): Arbeitsbeziehungen im Feld der Schulsozialarbeit - AdressatInnenperspektiven

- Monika Fey und Silke Birgitta Gahleitner (Alice Salomon Hochschule Berlin): »Das Umsetzen, das findet ja im Alltag statt « Kompetenzanforderungen an Fachkräfte der Sozialen Arbeit in stationären Jugendhilfesettings

Teil B: Ausbildung und Praxis (Moderation Silke B. Gahleitner)

- Winfried Effelsberg (Katholische Fachhochschule Freiburg): Klinische Soziale Arbeit mit psychisch kranken Menschen

- Claire Moore (Hochschule für Gesundheit Bochum): Planung eines Konzeptes für einen interprofessionellen und traumazentrierten MA-Studiengang der Klinischen Sozialarbeit

- Albert Mühlum (ehemals SRH Hochschule Heidelberg) und Hans Günther Homfeldt (Universität Trier): vom Adressaten zum Akteur/Aktor? Überlegungen zur Gesundheitsförderung und Krankheitsbewältigung im Kontext Klinischer Sozialarbeit: Konsequenzen für Ausbildung, Praxis, Kooperationen Davon wieder nur beispielhaft ein Auszug aus dem Abstract zum Vortrag von Bettina Schuhrke: »Durch eine Sekundärauswertung der Daten aus EVAS (»Evaluationssystem erzieherischer Hilfen «) ... wurde eine Einschätzung von psychischen Belastungen und Ressourcen von Kindern und Jugendlichen in stationären und teilstationären Erziehungshilfen gewonnen ... Die Ergebnisse betreffen das
Vorkommen von psychischen Problemen und diagnostizierten psychiatrischen Störungen, Ressourcen und psychosoziale Belastungen, Aufnahmeanlässe und bisherigen Jugendhilfekarrieren: Kinder und Jugendliche in den untersuchten Erziehungshilfen zeigen in hohem Maße psychische Auffälligkeiten. Die in dieser Studie besonders interessierenden Klienten mit problematischem sexuellem Verhalten erweisen sich als eine besonders belastete Gruppe. ... Diskutiert wird im Hinblick auf die vorliegenden Befunde, ob es für den Umgang mit besonders belasteten Kindern und Jugendlichen spezieller Kompetenzen bedarf, die über das im Rahmen von Bachelor-Studiengängen der Sozialen Arbeit vermittelte Methodenwissen hinausgehen und wie sich die Kooperation mit anderen Professionen mit spezialisiertem 'Störungswissen (insbesondere Mediziner, Psychologen) angesichts bestehender Weiterentwicklungen der Sozialen Arbeit gestalten könnte. «

\section{Podium und Plenumsdiskussion}

Am Podium vertreten waren: Prof. Dr. Peter Buttner, Präsidiumsmitglied Deutscher Verein für öffentliche und private Fürsorge e.V.; Prof. Dr. Silke Birgitta Gahleitner, Deutsche Gesellschaft für Soziale Arbeit e. V.; Michael Leinenbach, Deutscher Berufsverband für Soziale Arbeit; Prof. Dr. Ulrich Bartosch, Vorsitzender Fachbereichstag Soziale Arbeit, Fabian Kessel, Vorstandsmitglied der Kommission Sozialpädagogik in der Deutschen Gesellschaft für Erziehungswissenschaft.

Die Moderation lag bei Prof. Dr. Sabine Stövesand, Vorstandsmitglied der Deutschen Gesellschaft für Soziale Arbeit. Die Moderatorin legte jedem Podiumsteilnehmer eine Leitfrage vor, die nach dessen Statement plenumsoffen diskutiert wurde.

- Zu Spezialisierungen in der Hochschullandschaft nahm Peter Buttner Stellung. Binnendifferenzierung und Außendifferenzierung stehen für ihn in einem Zusammenhang. Die Vielfalt der Spezialisierungen auf Masterniveau sei sowohl dem gesellschaftlichen Problemdruck wie dem Profilierungsdruck der Hochschulen nach Bologna geschuldet - mit Vorzügen und Problemen. Diese wurden lebhaft erörtert.
Beispiel: Da es schon immer Vertiefungsbereiche im Studium gab, sei die heutige Master-Spezialisierung nur konsequent. Dem wurde entgegnet, die Praxistauglichkeit fehle weitgehend. Ebenso kontrovers wurde das Verhältnis von Wissenschaft und Ausbildung sowie Wissenschaft und Praxis diskutiert.

- Zu Chancen und Gefahren des Differenzierungsprozesses führte Silke Gahleitner aus, Sozialarbeit müsse auf Bachelor-Level generalistisch sein, dies sei aber auch nur begrenzt möglich. Daher sei eine anschließende Spezialisierung mit höherer Anforderungsstruktur nötig. Leider gehe dabei oft die Identität als Sozialarbeiterin oder Sozialarbeiter verloren, Beispiel Therapiebereich: Bei aller notwendigen Vielfalt wird ein gemeinsamer Kern oder Rahmen für nötig gehalten, auch als »disziplinäres Band $«$ in der Außenwirkung. Kritisch gefragt wird, ob die Dichotomie »Generalist vs. Spezialist « überhaupt zutreffe und ob nicht eher über Schnittflächen und neue Koalitionen nachgedacht werden müsste.

- Bezüglich Ausgestaltung und praktischen Auswirkungen plädiert Michael Leinenbach für ein Berufsgesetz (ersatzweise für ein Berufsregister) in Verbindung mit Berufsethos und Schlüsselkompetenzen. Er argumentiert für eine generalistische Ausbildung und stärkere Einbeziehung der Praxis (Praxisjahr). Hier flammt die Kontroverse Wissenschaft, Ausbildung und Praxis erneut auf: Kann, will, soll die Hochschule für Praxis ausbilden und falls ja, unter welchen Bedingungen?

- Als hochschulpolitische Konsequenz fordert Ulrich Bartosch, die Studiengänge der Sozialen Arbeit müssten sich zuerst als hochschulische Bildung profilieren, da die Orientierung an Zweckrationalität (Praxiserfordernisse) Brüche bewirke. Dagegen wird argumentiert, professionelle Ausbildung brauche das Zusammenwirken verschiedener Funktionsbereiche (beispielhaft: Zusammensetzung des Podiums), die an einem Konzept oder einer »Denkfigur « arbeiten könnten, zu dem ein gemeinsamer Kern und permanente Reflexionsfähigkeit gehören.

- Zur Perspektive - »Was tut Not? « warnt Fabian Kessl vor einer verbindlichen Festlegung von Inhalten im Sin- 
ne eines Kerncurriculums. Dieses sei in der Erziehungswissenschaft grandios gescheitert, da in den Curricula nicht umgesetzt. Auch die »Bezugswissenschaften « seien keineswegs » einheitlich «, sondern höchst heterogen. Im Übrigen vertrage sich dies nicht mit der Funktion von Wissenschaft. Dem wird entgegnet, Wissenschaft habe doch die Aufgabe und Chance, zur Problemerkennung und Problemlösung beizutragen, wie auch die rege Forschungstätigkeit der Hochschulen zeige.

Auf der Suche nach Perspektiven für Ausbildung und Selbstbild der Sozialen Arbeit wird im weiteren Verlauf eine Profilmatrix vorgeschlagen, die sich am Qualifikationsrahmen des Fachbereichstags orientieren könnte oder an der Modulanalyse von Wilhelm Klüsche. Jedenfalls seien Standards und Grundprämissen nötig, außerdem müsse die Breite der Sozialen Arbeit abgebildet sein und Offenheit für Veränderungen bestehen. Vermutlich ist der Spannungsbogen von Kernbestand und variablen Elementen sogar konstitutiv, wie die Geschichte zeigt. Leider kamen weitere Stimmen aus dem Plenum nur noch ansatzweise zu Wort, dennoch bleibt ein positives Fazit.

\section{Fazit}

Das Thema »Generalisierung und Spezialisierung " traf den Nerv der Zunft, zumal Ganzheitlichkeit zum Topos der Sozialarbeit gehört, aber vor neuen Herausforderungen steht. Auch wenn es keine abschließenden Ergebnisse gab und geben konnte- die Bemühungen lassen sich im Nachhinein als Versuche zur Konstruktion und Dekonstruktion von Ganzheit interpretieren -, so ist die Debatte doch notwendig.

Die Stärke der Veranstaltung lag darin, die Positionen im Gespräch zu halten und so unterschiedliche Institutionen und Repräsentanten, Akteure und Stakeholder der Sozialen Arbeit wie bei diesem Podium zusammenzubringen. Die Jahrestagung steht dabei in der Kontinuität der Deutschen Gesellschaft für Soziale Arbeit, die sich immer mehr zum Zentrum der wissenschaftlichen Gemeinschaft der Lehrenden und Forschenden entwickelt. Wie die Panels anschaulich demonstrierten, ist dies vor allem den Sektionen und Fachgruppen zu verdanken, die den wissenschaftlichen Nachwuchs ansprechen, fordern und fördern.

\section{Was fasst man eigentlich unter die Sammelbezeichnung »Soziale Berufe«?}

Soziale Berufe (gelegentlich auch: Sozialberufe) ist ein Sammelbegriff für eine gegenüber anderen Berufsgruppen nicht eindeutig abgegrenzte Gruppe von Berufen im weiteren Sinne, die mit sozialen und teils pflegerischen Dienstleistungen, sozialer Hilfe und Kontrolle, Beratung, Erziehung, Betreuung und Unterstützung und verwandten Formen personennaher Tätigkeiten im Sozial-, Gesundheits- und Bildungswesen, teilweise auch im Justizwesen und der Arbeitsverwaltung betraut sind. Durch ihre professionalisierte Kommunikations-, Vermittlungs- und Organisationsarbeit helfen die sozialen Berufe Leid zu mindern, Not abzuwenden, persönliche Entwicklung und gemeinschaftlichen Zusammenhalt $\mathrm{zu}$ fördern und schließlich auch die soziale Ordnung zu stabilisieren. Soziale Berufe üben damit elementare zwischenmenschliche, gemeinschaftliche und gesellschaftliche Funktionen aus.

Berufssystematisch werden die sozialen Berufe den Dienstleistungsberufen zugerechnet. Nach der vom Statistischen Bundesamt 1992 herausgegebenen »Klassifizierung der Berufe « stellen die sozialen Berufe zusammen mit den Lehrer/innen, den geistes- und einigen naturwissenschaftlichen Berufen sowie den »Berufen in der Seelsorge « eine Untergruppe der Dienstleistungsberufe dar. Die Gruppe der sozialen Berufe selbst (Berufsgruppe 86) umfasst im Einzelnen: Sozialarbeiter/innen und Sozialpädagog/innen (Berufskennziffer 861), Heilpädagog/innen (Heilpädagogik) (862), Erzieher/innen (863), Altenpfleger/innen (864), Familienpfleger/innen und Dorfhelfer/ innen (865), Heilerziehungspfleger/ innen (866), Kinderpfleger/innen (867), Arbeits- und Berufsberater/innen (868) sowie eine Restkategorie »sonstige soziale Berufe« (869).

Ausschlaggebendes Kriterium der Systematik in der »Klassifizierung der Berufe « 1992 ist die Art der beruflichen Tätigkeit, jedoch nicht Status, Rangordnung, Qualifikationsniveau oder berufliche Stellung. Demzufolge fallen beispielsweise unter die Kennziffer 861 gleichermaßen Absolvent/innen von
Hochschulen und Fachschulen bis hin zu ungelernten Kräften. Abgesehen von der Altenpflege sind die Pflegeberufe nicht bei den sozialen Berufen, sondern in der Berufsgruppe 85 als Untergruppe der Gesundheitsdienstberufe aufgeführt. Nachdem jedoch die Altenpflege in der berufsrechtlichen Verortung, den Ausbildungsstrukturen und auch in ihrem Selbstverständnis an die Gesundheits- und Krankenpflege herangerückt ist, kann die uneinheitliche Zuordnung der Pflegeberufe in der "Klassifizierung der Berufe « nicht mehr als konsistent gelten. Derzeit ist eine Neufassung der Klassifizierung der Berufe in Vorbereitung, bei der diese und andere Inkonsistenzen bereinigt werden sollen. Es soll aber auch der Anschluss an die internationale Berufestatistik ISCO gewährleistet werden. Für die »Klassifizierung der Berufe « 2010 erfolgt daher eine Neusystematisierung, die neben der berufsfachlichen Dimension (verrichtete Tätigkeiten) die Dimension des Anforderungsniveaus erfasst. Insofern folgt die »Klassifizierung der Berufe « 2010 dem Trend zur Hierarchisierung im Bildungs- und Beschäftigungssystem, wie er in der europäischen Bildungspolitik seit ca. zehn Jahren deutlich wird.

In der mit 29.10.2009 durch die Europäische Kommission auf den Weg gebrachten »International Standard Classification of Occupations « ISCO-08, die von den Mitgliedsstaaten der Europäischen Union ab dem Jahr 2011 »in allen statistischen Bereichen, in denen nach Berufen aufgegliederte Statistiken bereitgestellt werden « verwendet werden soll (Empfehlung 2009/824/EG), sind die Berufe und Tätigkeiten nicht in berufsfachlich gegliederte Gruppen, sondern primär nach Tätigkeits- und Qualifikationsniveau aufgeschlüsselt. Die sozialen Berufe sind hier also nicht in einer Gruppe zusammengefasst, sondern ziehen sich, wie andere Berufsgruppen auch, als ein loser Strang durch die weitgehend hierarchisch geschichteten Hauptgruppen: von den »Führungskräften in der Erbringung von Dienstleistungen der Sozialfürsorge « über die akademisch ausgebildeten Sozialarbeiter/ innen und die »nicht akademischen sozialpflegerischen Fachkräfte» bis zu verschiedenen 
sozialen Berufen auf niedrigem Qualifikationsniveau wie z. B. Kinderbetreuer/innen, Haus- und Familienpfleger/innen, Pflegehelfer/innen.

Rechtlich gesehen unterscheiden sich die sozialen Berufe von den Heilberufen durch die gesetzgeberische Zuständigkeit: Die Heilberufe unterliegen nach Art. 74 Abs. 1 Grundgesetz (GG) dem Bundesrecht, die sozialen Berufe dem Landesrecht. Bundesrechtlich geregelt sind demnach u. a. die Berufe der Physiotherapie, Ergotherapie und Logopädie, der Gesundheits- und Kranken- sowie Kinderkrankenpflege. Mit Urteil des Bundesverfassungsgerichts vom 24.10.2002 ist auch die Altenpflege ein »anderer « (d. h. nicht ärztlicher) Heilberuf im Sinne des Art. 74 GG geworden. Dagegen unterliegen die Helferberufe, auch die der Alten- und Krankenpflegehilfe, der Regelungskompetenz der Länder, gleichermaßen die Heilpädagog/innen und Heilerziehungspfleger/ innen. $\mathrm{Zu}$ den landesrechtlich geregelten sozialen Berufen gehören ferner die Erzieher/ innen. Das Tragen der Berufsbezeichnung »staatlich anerkannter Erzieher/staatlich anerkannte Erzieherin « ist nach Vereinbarung der Kultusministerkonferenz vom 28.1.2000 an die erfolgreich abgeschlossene Ausbildung und meist weitere im Landesrecht festgelegte Voraussetzungen gebunden (ähnlich: Heilpädagogik). Die staatliche Anerkennung für Sozialarbeiter/ innen und Sozialpädagog/innen mit Diplom- oder Bachelor-Abschluss ist in vielen Bundesländern Voraussetzung für die Berufsausübung. $\mathrm{Zu}$ den rechtlichen Regelungen zählt auch das Fachkräftegebot für die Kinderund Jugendhilfe im $\mathbb{S} 72$ Sozialgesetzbuch (SGB) VIII, das aber nur abstrakte Vorgaben macht und keine einzelnen Berufe bzw. Ausbildungen als Voraussetzung für den Fachkräftestatus nennt (ähnlich auch $\mathbb{} 6$ SGB XII) (Ausbildung in sozialen Berufen).

Eine abschließende Auflistung der sozialen Berufe erscheint wegen der Begriffsunschärfen und Inkonsistenzen sowie in Anbetracht des dynamischen Wandels im Bildungssystem nicht sinnvoll. Es lassen sich jedoch drei nach Umfang gestaffelte Begriffsvarianten herausarbeiten: In der Variante mit dem engsten Begriffsumfang sind nur die zentralen sozialpädagogischen Berufe enthalten, also die in der Sozialen Arbeit mit Fachhochschul- oder Universitätsabschluss Tätigen sowie die Erzieher/innen und Kinderpfleger/innen. Die nächstweitere Variante umfasst darüber hinaus die Heilpädagog/innen, Heilerziehungspfleger/ innen, Erzieher/innen und die in spezialisierten Pädagogikfeldern Tätigen (ohne Lehrer/innen) sowie die Altenpflege, soweit sie nicht primär auf körperliche Pflege ausgerichtet ist. Diese mittlere Begriffsvariante ist die gängigste. In der Variante mit dem weitesten Begriffsumfang kommen noch hinzu: die Pflegeberufe, die Gesundheitsberufe (auch Diätassistent/innen, Motopädagog/innen), die seelsorgerischen und die Beratungsberufe (Beratung) sowie in beschränktem Umfang Bildungsberufe (Lehrer/innen). Zivildienst und Freiwilliges Soziales Jahr, deren Tätigkeitsspektrum sich mit denen einfacher sozialer Berufe teilweise deckt, werden nicht zu den sozialen Berufen gezählt, weil es sich nicht um berufliche Tätigkeiten handelt.

Die sozialen Berufe waren seit ihren Anfängen im 19. Jahrhundert Frauenberufe und sind dies bis heute geblieben. Der Name »soziale Frauenschulen « belegt die weibliche Ausrichtung für die Gründerzeit der beruflichen Ausbildung in der
Sozialen Arbeit vor ca. 100 Jahren. Diese soziale Berufstätigkeit entsprang zu guten Teilen der bürgerlichen Frauenbewegung. Sie stellte für Frauen eine der wesentlichen Eintrittspforten in die Arbeitswelt dar. Heute sind die akademischen sozialen Berufe typische Aufstiegsberufe, insbesondere für Frauen. Nach Angaben des Statistischen Bundesamtes waren im Jahr 2008 von den in sozialen Berufen Erwerbstätigen 83 Prozent Frauen, wobei deren Anteil bei den Erzieher/innen $(92 \%)$ am höchsten und in der Sozialarbeit/Sozialpädagogik $(68 \%)$ am niedrigsten ist. Bei den Führungskräften sind allerdings überproportional Männer anzutreffen. Heute spielt für den hohen Frauenanteil auch die Tatsache eine Rolle, dass viele Stellen in Teilzeit angeboten werden. Die überwiegende Mehrzahl, der in den sozialen Berufen Tätigen, arbeitet im Angestelltenverhältnis. In der Sozialarbeit/ Sozialpädagogik gibt es auch einen kleinen Prozentsatz an Beamten und Selbstständigen.

Soziale Berufe sind mit der funktionalen Differenzierung der Gesellschaft entstanden. Was in der traditionellen Fürsorge (Geschichte der sozialen Arbeit) von Laien oder Ordensleuten erbracht wurde, liegt heute z. T. in den Händen spezialisiert ausgebildeter Fachkräfte, deren Handeln durch professionelle Traditionen oder explizite Professionalisierungsstrategien vorgezeichnet, durch gesetzliche Vorgaben gebunden und durch Wissenschaftlichkeit sowie sozialberufliche Ethik-Kodizes (Ethik in der sozialen Arbeit) legitimiert ist. Zwar ist das nicht professionelle Helfen (Hilfe/Helfen) in Form von Betreuung durch Angehörige, gegenseitiger Hilfe, privat erbrachter Pflege, ehrenamtlicher Tätigkeit etc. nach wie vor ein elementar wichtiger Teil der in der Summe geleisteten Hilfen und damit komplementär zu Funktion und Leistungen der sozialen Berufe, jedoch sind die sozialen Berufe nicht durch freiwillige Hilfen und Ehrenamt ersetzbar (Ehrenamtliche/freiwillige Tätigkeit im sozialen Bereich). Sowohl wegen des absehbaren Fachkräftemangels als auch aufgrund zivilgesellschaftlicher Überlegungen ist es für beide Seiten wichtig, immer wieder Formeln für die ergänzende und kooperierende Zusammenarbeit auszuhandeln. Dazu gehört auch die Qualifizierung des Ehrenamts. Die Abgrenzungsbemühungen seitens der sozialen Berufe gegenüber nicht professionellen Hilfen können als ein Aspekt des Professionalisierungsstrebens interpretiert werden, tatsächlich aber stellen Anleitung und Qualifizierung des Ehrenamtes eine eigene professionelle Herausforderung und Aufgabe für die sozialen Berufe dar.

Die zunehmende Ausdifferenzierung sozialprofessioneller Tätigkeiten und ihrer Ausbildungen und die Konkurrenzen um berufliche Claims erfordern auch eine erhöhte Aufmerksamkeit für die Formen der Kooperation zwischen den einzelnen sozialen Berufen. Dies gilt umso mehr, je weiter sich die sozialen Berufe in der Vertikale (hierarchisch) wie auch Horizontale (Spezialisierungen) weiter diversifizieren. Die akademischen Ausbildungen haben sich mit dem Bolognaprozess und den Hochschulstrukturreformen vervielfacht, insbesondere im Segment der Masterstudiengänge (Bachelor-Master-System): Auf diesem Wege haben sich z. B. das Sozialmanagement als akademischer Beruf mit enger Beziehung zu den sozialen Berufen und die Klinische Sozialarbeit als Spezialisierung innerhalb der Sozialen Arbeit etabliert. Im Segment der Bachelorstudiengänge sind es v. a. die Studiengänge zur Erziehung und 
Bildung (akademisierte Erzieher/innenausbildung). Die Diversität der Berufe nimmt aber auch am unteren Ende der Qualifikationsskala zu: Die »Fachkraft für Gesundheits- und Sozialdienstleistungen « beruht auf einem Zertifikatslehrgang, der Pflegehelfer/innen die »Mitarbeit im Haushalt und der täglichen Versorgung « erleichtern soll. Für die Kindertagespflege erfolgt auf Basis eines 160-Stunden-Curriculums eine Grundqualifizierung von Tagespflegepersonen.

Der Bedarf an sozialen Dienstleistungen und der Mengenzuwachs an Beschäftigten hängen mit der Entwicklung des Wohlfahrtsstaates und der Etablierung sozialer Rechte zusammen. Die Beschäftigtenzahl ist seit den 1950er-Jahren drastisch gestiegen und für die nahe bis mittlere Zukunft wird ein Fachkräftemangel prognostiziert. Die Geschichte der sozialen Berufe ist also auch eine Geschichte ihrer Expansion, wenngleich die Zuwächse an beschäftigten Personen größer sind als die Zuwächse an Arbeitsvolumen, also an Umfang geleisteter Stunden. Im Jahr 2009 waren ca. 1,25 Mio. Personen sozialversicherungspflichtig in den sozialen Berufen beschäftigt (Beschäftigungsstatistik der Bundesagentur für Arbeit). Im Jahr 2005 waren es noch knappe 1,1 Mio. und 1995 nur ca. 810.000 Personen. Zählt man über die sozialversicherungspflichtig beschäftigten Personen hinaus auch alle sonstig Erwerbstätigen, also geringfügig Beschäftigte, mithelfende Familienangehörige etc., so waren im Jahr 2008 ganze 1,6 Mio. Personen in den sozialen Berufen erwerbstätig. Von diesen hatten 37 Prozent ein Abitur oder Fachabitur als höchsten Schulabschluss und 22 Prozent einen Hochschulabschluss von einer Fachhochschule oder Universität (Statistisches Bundesamt).

Die weitere Entwicklung der sozialen Berufe dürfte dynamisch bleiben, alleine schon, weil ein zunehmender Bedarf abzusehen und nicht klar ist, wie er zu decken ist. Zugleich stehen die sozialen Berufe unter einem steigenden Legitimationsdruck in puncto Effektivität ihres beruflichen Handelns (Effektestudien) sowie Transparenz der eigenen Leistungen.
Auch der entsprechende Qualifizierungsdruck hält an oder steigt. Beispiele für diesen erhöhten Qualifizierungsbedarf sind neben vielen anderen: haftungsrechtliche Fragen, evidenzbasiertes berufliches Handeln (evidence based social work), soziale Diagnostik, Umgang mit bzw. Nutzung von neuen Medien, Digitalisierung der sozialen Dienste. Was im Prinzip aber bestehen bleibt, ist die persönliche und institutionelle Verantwortung, die sich mit der Ausübung sozialen Berufe und dem damit privilegierten Zugang zur Privatsphäre der einbezogenen Menschen ergibt. Diese lässt sich durch formelhafte berufsethische Kodizes, wie sie heute verbreitet sind, nur sehr bedingt einfangen. Der Mehrzahl, der in den sozialen Berufen Tätigen, fällt somit die Aufgabe persönlicher moralischer Auseinandersetzung mit den beruflich entstandenen Fragen in besonderem Maße zu.

Peter Buttner

Prof. Dr. Peter Buttner ist Hochschullehrer an der Fakultät für angewandte Sozialwissenschaften der Hochschule München und Präsidiumsmitglied Deutscher Verein für öffentliche und private Fürsorge e.V.

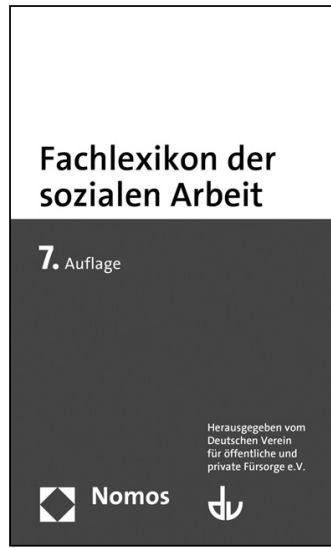

Quelle: Deutscher Verein für öffentliche und private Fürsorge e. V. (Hg.): Fachlexikon der sozialen Arbeit. 7. Auflage. Nomos Verlagsgesellschaft, Baden-Baden 2011. Ca. 1.200 Seiten. 44,- Euro. ISBN 9783-8329-5153-5. Seite $783 \mathrm{ff}$.

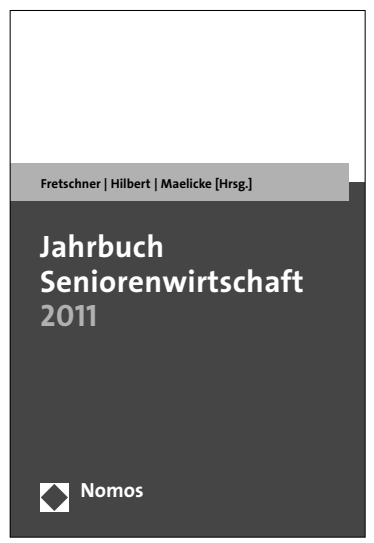

Bitte bestellen Sie im Buchhandel oder versandkostenfrei unter $\downarrow$ www.nomos-shop.de

\begin{abstract}
Jahrbuch Seniorenwirtschaft 2011
Herausgegeben von Prof. Dr. Rainer Fretschner, PD Dr. Josef Hilbert und Prof. Dr. Bernd Maelicke

2011, ca. 200 S., brosch., ca. 39,- €, ISBN 978-3-8329-6140-4

Erscheint ca. Mai 2011

Die Seniorenwirtschaft gehört weltweit zu den Branchen mit den größten Zuwachsraten. Auch in Deutschland wird der demographische Wandel immer mehr als Herausforderung für Politik, Wirtschaft und Wissenschaft verstanden. Im vorliegenden Jahrbuch analysieren nationale und internationale Experten Trends und Megatrends dieses Entwicklungsprozesses.
\end{abstract}

